UCLA/97/TEP/20

IEM-FT-163/97

hep-ph/9709289

Revised Version April 98

\title{
Generation of Large Lepton Asymmetries
}

\author{
Alberto Casas ${ }^{\mathrm{a}}$, Wai Yan Cheng ${ }^{\mathrm{b}}$, Graciela Gelminic ${ }^{\mathrm{c}}$ \\ anstituto de Estructura de la Materia, CSIC \\ Serrano 123, 28006 Madrid, Spain \\ casas@cc.csic.es \\ ${ }^{\mathrm{bc}}$ Department of Physics and Astronomy, University of California, UCLA \\ 405 Hilgard Ave., Los Angeles, CA 90095 \\ bwcheng@physics.ucla.edu, \\ c gelmini@physics.ucla.edu
}

\begin{abstract}
We present here a realistic model to produce a very large lepton asymmetry $L \simeq$ $10^{-2}-1$, without producing a large baryon asymmetry. The model is based on the Affleck and Dyne scenario, in which the field acquiring a large vacuum expectation value during inflation is a s-neutrino right. We require $L$ to be large enough for the electroweak symmetry to be spontaneously broken at all temperatures after inflation, with the consequent suppression of sphaleron transitions.
\end{abstract}

\footnotetext{
${ }^{1}$ Research supported in part by: the CICYT (contract AEN95-0195) and the European Union (contract CHRX-CT92-0004) (JAC); and the US Department of Energy under grant DE-FG03-91ER40662 Task C (GG).

${ }^{2}$ On leave at CERN (Theory Division) until June 15, 1998.
} 
The possible role of neutrino degeneracy in nucleosynthesis has been studied several times from 1967 onwards [1] [2]. A large number of electron neutrinos, $\nu_{e}$, present during nucleosynthesis yields a reduction of the neutron to proton ratio $n / p$, through the reaction $n \nu_{e} \rightarrow p e$. This in turn lowers the ${ }^{4} \mathrm{He}$ abundance, since when nucleosynthesis takes place essentially all neutrons end up in ${ }^{4} \mathrm{He}$ nuclei. Extra neutrinos of any flavor would increase the energy density of the Universe, leading to an earlier decoupling of weak interactions and consequent increase of $n / p$ (and ${ }^{4} \mathrm{He}$ ). This last effect is less important than the former one in the case of electron neutrinos, but it is the only effect of an excess of muon and/or tau neutrinos, $\nu_{\mu}$ and $\nu_{\tau}$. Thus when both the chemical potentials of $\nu_{e}$ and of $\nu_{\mu}, \nu_{\tau}$ are large, their effect largely compensate each other. This allows to obtain light element abundances in agreement with observations even for values of the baryon to photon ratio $\eta \equiv n_{B} / n_{\gamma}$ much larger than in standard nucleosynthesis, which is $\eta \simeq O\left(10^{-10}\right)$. Therefore, the usually quoted nucleosynthesis upper bound on the cosmological density of baryons $\Omega_{B}$ may be falsified and much larger values of $\Omega_{B}$ become allowed by nucleosynthesis, even $\Omega_{B}=1$ (which corresponds to $\eta \simeq O\left(10^{-8}\right)$ ).

More restrictive upper bounds on $\Omega_{B}$ can be obtained, however, by examining other consequences of a large lepton number [3]. Due to the electric charge neutrality of the Universe, the excess of protons with respect to antiprotons must be accompanied by the same excess of electrons over positrons, so $L_{e \pm} \equiv\left(n_{e}-n_{\bar{e}}\right) / s \simeq B \equiv\left(n_{B}-\right.$ $\left.n_{\bar{B}}\right) / s \simeq O\left(10^{-10}-10^{-8}\right)$. A larger lepton number can reside only in neutrinos. We will be interested in values of $L=L_{e}+L_{\mu}+L_{\tau}$ of order one. Thus to a very high accuracy $L_{\alpha}=\left(n_{\nu_{\alpha}}-n_{\bar{\nu}_{\alpha}}\right) / s$, where $\alpha=e, \mu, \tau$. Here $n$ stands for the number density of the particle indicated in the suffix and $s$ is the entropy density of the Universe. The asymmetries $L_{\alpha}$ are related to the chemical potentials, $\mu_{\alpha}$,

$$
L_{\alpha}=\frac{45}{12 \pi^{4} g_{s *}\left(T_{\gamma}\right)}\left(\frac{T_{\nu}}{T_{\gamma}}\right)^{3}\left(\pi^{2} \xi_{\alpha}+\xi_{\alpha}^{3}\right) \simeq 3.6\left(10^{-2} \xi_{\alpha}+10^{-3} \xi_{\alpha}^{3}\right)
$$


where $\xi_{\alpha} \equiv \mu_{\nu_{\alpha}} / T_{\nu}$ are dimensionless chemical potentials, $g_{s *}$ is the $T_{\gamma}$ dependent entropy number of degrees of freedom, i.e. $s=\left(2 \pi^{2} / 45\right) g_{s *} T_{\gamma}^{3}=\pi^{4} g_{s *} n_{\gamma} / 45 \zeta(3) \simeq 1.8008 g_{s *} n_{\gamma}$, and the numerical relation holds during nucleosynthesis. Notice that, with only the relativistic particles in the standard model present during nucleosynthesis, the relation between the temperature of neutrinos, $T_{\nu}$, and the temperature of photons, $T_{\gamma}$, is given by $g_{s *}\left(T_{\gamma} / T_{\nu}\right)^{3}=10.75$, both before and after $e^{+} e^{-}$annihilation, what means $T_{\nu}=T_{\gamma}$ before $e^{+} e^{-}$annihilation. In the presence of large neutrino asymmetries this relation holds only if $\xi_{\alpha}<12$ [2]. For larger $\xi_{\alpha}$, instead, $T_{\nu}$ would be lower, since the neutrino decoupling temperature becomes larger than the muon mass [2]. After neutrino decoupling, $L_{\alpha}$ and $T_{\nu}^{3} / s$ are constant, and so are the $\xi_{\alpha}$.

The energy density of stable relativistic neutrinos,

$$
\rho=\sum_{\alpha=e, \mu, \tau} \frac{\pi^{2}}{15} T_{\nu}^{4}\left[\frac{7}{8}+\frac{15}{4 \pi^{2}}\left(\xi_{\alpha}^{2}+\frac{\xi_{\alpha}^{4}}{2 \pi^{2}}\right)\right],
$$

leads to an upper bound on the $\xi_{\alpha}$ due to the limit on the present total energy density of the Universe (in units of the critical density), $\Omega_{o}$. The bound is $\xi_{e}+\xi_{\mu}+\xi_{\tau}<$ 86 for $\Omega_{o} h^{2} \lesssim 1 / 4$, as required if $\Omega_{o} \leq 1$ and $t_{o} \gtrsim 10^{10} \mathrm{yr}$ in a radiation dominated Universe [2] ( $h$ is the Hubble constant in units of $100 \mathrm{~km} / \mathrm{sec} \mathrm{Mpc}, h=0.4-1$ and $t_{o}$ is the present age of the universe). However, galaxy formation arguments provide a stronger upper limit. Stable relativistic neutrinos in the large numbers considered here would maintain the Universe radiation dominated (by the neutrinos) for much longer (thus until lower temperatures) than in the standard cosmology. Requesting neutrinos to become subdominant before the recombination epoch, Kang and Steigman [2] found, using nucleosynthesis bounds, $-0.06 \lesssim \xi_{\nu_{e}} \lesssim 1.1,\left|\xi_{\nu_{\mu}, \nu_{\tau}}\right| \lesssim 6.9, \eta=n_{B} / n_{\gamma} \lesssim 19 \times 10^{-10}$, and, therefore, $\Omega_{B} h^{2}=\left(\eta / 272.2 \times 10^{-10}\right) \lesssim 0.069$. This is an upper bound on $\Omega_{B}$ almost an order of magnitude larger than obtained in conventional nucleosynthesis. This would, for example, provide a solution to what some call "the x-ray cluster baryon crisis" [⿴囗十 [ [5]. 
In fact the conventional nucleosynthesis bound on $\Omega_{B}$ combined with the measurement of a "large" amount of gas in rich clusters of galaxies leads to an upper bound on the amount of dark matter (DM) in the Universe, i.e. $\Omega_{D M} \leq(0.2-0.4) h^{-1 / 2}$ 四. If $h>0.16$, as all measurements confirm, this would mean $\Omega_{D M}<1$, namely that we either live in an open Universe or in a Universe with a cosmological constant. Since the upper bound just mentioned on $\Omega_{D M}$ is proportional to the nucleosynthesis upper bound on $\Omega_{B}$, making the latter one order of magnitude larger would eliminate this "crisis". Notice that using Eq. (1), the Kang and Steigman the bound $\xi_{\alpha} \lesssim 6.9$ translates into $L_{\alpha} \lesssim 1.4$.

The production itself of a very large $L$ is problematic. In the standard out-ofequilibrium decay scenario for the generation of the baryon asymmetry both $B$ and $L$ are typically small. The largest asymmetries produced in this scenario are of order $L \simeq \epsilon\left(n_{X} / g_{s *} n_{\gamma}\right)$, where $n_{X}$ is the number density of decaying particles and $\epsilon$ is the CP-violating parameter that gives the lepton number generated per decay. Taking the generic value $g_{s *} \gtrsim 10^{2}$ at early times, we see that $\epsilon=1$ and $n_{x}=n_{\gamma}$ are necessary to get at most to $L \simeq 10^{-2}$. Even if these values can be arranged for, they are not easy to obtain in realistic models [6]. Moreover, if the baryon and lepton number violating (but $B-L$ conserving) reactions due to sphalerons are in equilibrium after the production of an asymmetry $L$, they will result in $B \simeq-L$, implying that the total $L$ must be as small as $B$. Thus, if individual lepton numbers are large they should (almost) cancel each other (with a fine tuning of eight orders of magnitude if $\xi \simeq O(1)$ ).

Here we present a viable model, where naturally a large lepton number asymmetry can be generated, $L \simeq\left(10^{-2}-1\right)$, corresponding to $\xi_{\nu} \simeq O(1-10)$ (see Eq. (1)), namely, much larger, by seven to ten orders of magnitude, than the baryon asymmetry B. We use the most efficient mechanism to produce a large fermion number asymmetry, i.e. the decay of a scalar condensate carrying fermion number in a supersymmetric model, as first proposed by Affleck and Dine in 1985 [7]. Because we want to generate only a large 
$L$ (not a large $B$ also), we consider models with a s-neutrino condensate.

Most of the previous work dealing with the possibility of $L \gg B$ either [6] [8] did not take into account the conversion of $L$ into $B$ due to sphaleron mediated processes before the electroweak phase transition [9] (for temperatures $M_{W} / \alpha_{w}>T>M_{W}$ ), or [10] did not actually dealt with the production of a large L. Only the following two potentially viable type of models have being so far proposed, to our knowledge, both very different from the model we present here. One of them is due to Dolgov and Kirilova [1]. They pointed out that a large $L$ and small $B$ could result within the Affleck and Dyne scenario, if very different rates of particle production (due to oscillations in field directions perpendicular to each flat direction) could be arranged for the $L$ and $B$ carrying flat directions, through a choice of coupling constants. Since particle production depletes the charge stored in the condensate, they suggested that the initially large $B$ charge might be efficiently dumped by particle production and not the large $L$ charge. Secondly, models where a large $L$ asymmetry could be generated at low temperatures due to neutrino-antineutrino oscillations have been proposed [12] recently.

Our model is based on the Affleck and Dyne mechanism to generate a large $L$ and on the idea, proposed by Linde twenty years ago [13], that, in the presence of a large enough lepton asymmetry $L>L_{C}$, the electroweak symmetry is never restaured at large temperatures, and, therefore, sphalerons are suppressed at all temperatures. The necessary critical value $L_{C}$ depends on the standard Higgs field mass. For Higgs masses of $60 \mathrm{GeV}$ to $1 \mathrm{TeV}$ the critical values of $n_{\nu} / n_{\gamma}$ needed at $T>M_{W}$ range from 2.4 to 13.3 [10]. Using the relation $s=1.80 g_{s *} n_{\gamma}$ and considering that a typical value of $g_{s *}$ at $T>M_{W}$ is $\sim 100$, gives $L_{C} \simeq(1.3$ to 7.4$) 10^{-2}\left(100 / g_{s *}\left(T>M_{W}\right)\right)$. Thus, if $L>L_{C}$ the weak gauge bosons are always massive and, consequently, the rate of sphaleron reactions is always much smaller than the rate of expansion of the Universe. Therefore $B$ and $L$ would be preserved [14], or at most a very small fraction of a large $L$ could be converted 
into $B$ by out of equilibrium sphaleron reactions, giving origin to the small $B$ observed (if no larger $B$ was produced earlier) [10. Considering the bound from galaxy formation mentioned above, we are interested in generating an $L$ in the range $L_{C}<L<1.4$.

The particular particle model we study is the Minimal Supersymmetric Standard Model supplemented with three electroweak singlet right handed neutrino superfields $N_{i}$, $i=1,2,3$ (whose scalar components will be denoted $\tilde{N}_{i}$ in what follows) and supersymmetric masses $M_{i}$. We take the $N_{i}$ mass terms to be diagonal for simplicity. Thus, besides the terms of the MSSM, this model has in the superpotential

$$
W=\frac{1}{2} M_{i} N_{i}^{C} N_{i}^{C}+h_{i j} N_{i}^{c} L_{j} H_{u}
$$

There is also a separate hidden inflationary sector, not leading to preheating [15], that we do not need to specify beyond giving the inflaton mass $m_{\psi}$ at the end of inflation and the inflaton decay rate $\Gamma_{\psi}$, for which we only use that the inflaton coupling is gravitational (i.e. $\left.g_{\psi} \simeq m_{\psi} / M_{P}\right)$, thus $\Gamma_{\psi} \simeq m_{\psi}^{3} / M_{P}^{2}\left(M_{P}\right.$ is the Planck mass, $\left.1.2 \times 10^{19} \mathrm{GeV}\right)$. We assume the see-saw mechanism is responsible for the mass of the light (mostly lefthanded) neutrinos, with hierarchical right handed Majorana masses $M_{1}<M_{2}<M_{3}$ and Dirac neutrino masses $m_{i j}^{D} \simeq h_{i j} v_{u}$ where $v_{u}=\left\langle H_{u}\right\rangle \simeq 10^{2} \mathrm{GeV}$ (so that $M^{D}$ has eigenvalues $m_{1}^{D}, m_{2}^{D}$ and $m_{3}^{D}$ and, if $\nu_{1}$ is the lightest and $\nu_{3}$ the heaviest neutrino, for example, then $\left.m_{1}^{D}<m_{2}^{D}<m_{3}^{D}\right)$.

We assume that $\tilde{N}_{1}$ is a flat direction of the potential during inflation. This implies that $M_{1}$ is smaller than the expansion rate of the Universe during inflation, $M_{1}<H_{\text {inf }}$. In this case, through different possible mechanisms that we will explore later, the field $\tilde{N}_{1}$ may find itself at the end of inflation with a non-zero value, say $\tilde{N}_{o}$. Let us examine first how large $\tilde{N}_{o}$ needs to be, before studying the mechanisms that naturally can lead to the range of values needed.

The sequence of events we envision is the following (see Fig.1). 
$I$ )- The inflaton $\psi$ starts oscillating about the true minimum of its potential when the expansion rate of the Universe $H$ is $H_{I} \simeq m_{\psi}$. This requirement comes from the solution of the evolution of a classical field $\phi$ in an expanding Universe, $\ddot{\phi}+3 H \dot{\phi}+\partial V / \partial \phi=0$. Assuming $V(\phi) \simeq m^{2} \phi^{2}$, the solution is oscillatory only for $H<m$, while for $H>m$ the solution is overdamped and the field remains stuck at its initial value $\phi=\phi_{o}$. The energy in the inflaton oscillations redshifts like matter, so the universe becomes matter dominated (MD).

$I I)$ - Through the same arguments the $\tilde{N}_{1}$ sneutrino field oscillations start when $H=$ $H_{I I} \simeq M_{1}<H_{I} \simeq m_{\psi}$. We assume that at this point the inflaton energy density still dominates, $\rho_{\psi}>\rho_{\tilde{N}}$.

III)- The $3^{\text {rd }}$ event of our sequence, the decay of the inflaton, happens at a later time $t \simeq \Gamma_{\psi}^{-1} \simeq M_{P}^{2} / m_{\psi}^{3}$. Using the MD relation between $\mathrm{H}$ and $\mathrm{t}$, we obtain that $H_{I I I}=$ $(2 / 3) t^{-1} \simeq(2 / 3) \Gamma_{\psi}$. At this point the Universe becomes radiation dominated (RD) by the decay products of the inflaton.

$I V$ ) - The sneutrino $\tilde{N}_{1}$ decays later at $t \simeq \Gamma_{\tilde{N}}^{-1} \simeq\left(h^{2} M_{1} / 8 \pi\right)^{-1}$ and using the RD relation between $H$ and $t, H_{I V}=(2 t)^{-1} \simeq h^{2} M_{1} / 16 \pi$. Here $h$ is the largest $h_{1 j}$ coupling. This $h$ is also the Yukawa coupling constant that dominates the Dirac mass $m_{1}^{D} \simeq h v_{u}$. Thus, using $m_{\nu_{1}} \simeq h^{2} v_{u}^{2} / M_{1}$, we can eliminate the constant $h$ in favor of the lightest neutrino mass in what follows.

$V)$-Thermalization happens when the rate of interaction of particles in the Universe, $\Gamma_{\text {int }}$, becomes equal (and then larger) than the expansion rate of the Universe, i.e. when $H=H_{V}=\left(2 t_{t h}\right)^{-1} \simeq \Gamma_{\text {int }} \simeq n \sigma$, where $n$ is the number density of particles and $\sigma$ their typical cross section. The actual lepton asymmetry is either generated or released when the $\tilde{N}_{1}$ sneutrino decays. We require to generate in total an asymmetry $L>L_{C} \simeq$ $10^{-2}$. It is important that the $\tilde{N}_{1}$ decay occurs before thermalization is achieved. If so, thermalization happens in the presence of a large lepton asymmetry that breaks the 
electroweak symmetry from the beginning of the thermal bath and, consequently, the rate of sphalerons is always suppressed.

Let us examine the conditions for the previous sequence of events to be consistent. The measured anisotropy of the microwave background radiation requires $H_{\text {inf }}$ to be $10^{13}-10^{14} \mathrm{GeV}$ in most models (even if it may be lower, such as $10^{11} \mathrm{GeV}$, in some) [16]. If the inflaton potential during inflation is just quadratic (such as in chaotic inflation) then $m_{\psi} \simeq H_{\mathrm{inf}} / 3$ at the end of inflation (since $H_{\mathrm{inf}}^{2}=(8 \pi / 3) m_{\psi}^{2} \psi^{2} / M_{P}^{2}$ and generically $\left.\psi \simeq M_{P}\right)$. However, with more complicated potentials, $m_{\psi}$ can be smaller and still we can assume that the potential at the end of inflation is well approximated by $m_{\psi}^{2} \psi^{2}$. For example if $V(\psi)=\lambda \psi^{4}+m_{\psi}^{2} \psi^{2}$, and the quartic term dominates during inflation then $\lambda \simeq 10^{-13}$ [16]. Assuming that $\psi \simeq M_{P}$ during inflation and $m_{\psi} \simeq\left(10^{-7}\right.$ or $\left.10^{-8}\right) M_{P}$, the quadratic term in the potential would dominate as soon as $\psi$ became smaller than $M_{P}$ (by a factor of 3 or 30 ), at the end of inflation. There are some models in which $m_{\psi}$ is even lower, $m_{\psi}=10^{-9} M_{P}$ [17]. In what follows it will be important to have $m_{\psi} \lesssim 10^{12}$ $\mathrm{GeV}$, which is perfectly possible.

Notice that $H_{I I I} \equiv H\left(t=\Gamma_{\psi}^{-1}\right) \simeq 6 \mathrm{MeV}\left(m_{\psi} / 10^{12} \mathrm{GeV}\right)^{3}$ and we will be considering values of $M_{1}$ larger than $H_{I I I}$, so that $\tilde{N}_{1}$ starts oscillating before the inflaton decays $\left(H_{I I} \simeq M_{1}>H_{I I I}\right)$. In our scenario $\tilde{N}_{1}$ decays after the inflaton decays (namely $H_{I V}<$ $\left.H_{I I I}\right)$ if $\Gamma_{\psi} / \Gamma_{\tilde{N}}>1$. This requires

$$
M_{1}<1.6 \times 10^{8} \mathrm{GeV} \frac{\left(m_{\psi} / 10^{12} \mathrm{GeV}\right)^{3 / 2}}{\left(m_{\nu_{1}} / 10^{-4} \mathrm{eV}\right)^{1 / 2}} .
$$

The thermalization time and temperature, $t_{t h}$ and $T_{t h}$, are obtained by estimating the number density of particles $n$ and their cross section $\sigma$. We take $n \simeq P n_{\psi}$, where $P$ is the average number of daughter particles produced in a $\psi$-decay, and the interaction cross section to be of electromagnetic order $\sigma \simeq \alpha^{2} / E^{2}$. Here $E$ is the characteristic energy of the particles, that redshifted from a value $E=m_{\psi} / P$ at $t=\Gamma_{\psi}^{-1}$, thus $E=\simeq$ 
$\left(m_{\psi} / P\right)\left[a\left(t=\Gamma_{\psi}^{-1}\right) / a\left(t_{t h}\right)\right]$. Moreover, $n_{\psi}$ redshifts from a value $\rho_{\psi} / m_{\psi}$ at $t=\Gamma_{\psi}^{-1}$, thus $n_{\psi}\left(t_{t h}\right)=\left(\rho_{\psi}\left(t=\Gamma_{\psi}^{-1}\right) / m_{\psi}\right)\left[a\left(t=\Gamma_{\psi}^{-1}\right) / a\left(t_{t h}\right)\right]^{3}$ and $\rho_{\psi}\left(t=\Gamma_{\psi}^{-1}\right)$ is the critical density at $H=\frac{2}{3} \Gamma_{\psi}$, i.e. $\rho_{\psi}\left(t=\Gamma_{\psi}^{-1}\right) \simeq \Gamma_{\psi}^{2} M_{P}^{2} / 6 \pi$. We obtain $t_{t h}=\left(36 \pi^{2} M_{P}^{2} / \alpha^{4} P^{6} m_{\psi}^{3}\right)$. The essential condition $t_{t h}>\Gamma_{\tilde{N}}^{-1}$ requires (using $P=2$ )

$$
0.67 \times 10^{4} \mathrm{GeV} \frac{\left(m_{\psi} / 10^{12} \mathrm{GeV}\right)^{3 / 2}}{\left(m_{\nu_{1}} / 10^{-4} \mathrm{eV}\right)^{1 / 2}}<M_{1}
$$

Notice that Eqs. (4) and (5) leave always an interval of four orders of magnitude for the allowed values of $M_{1}$, independently of the values of $m_{\psi}$ and $m_{\nu_{1}}$. Computing the critical density $\rho_{t h}$ at $t_{t h}$, when $H=\left(2 t_{t h}\right)^{-1}$ and assuming that all that energy goes into radiation, $\rho_{t h}=\left(g_{*} / 3\right) T_{t h}^{4}$, we get the reheating temperature, that is very low

$$
T_{t h}=\frac{1}{34} \frac{\alpha^{2} P^{3} m_{\psi}^{3 / 2}}{g_{*}^{1 / 4} M_{P}^{1 / 2}}=2.3 \times 10^{3} \mathrm{GeV}\left(\frac{\alpha}{10^{-2}}\right)^{2}\left(\frac{P}{2}\right)^{3} \frac{\left(m_{\psi} / 10^{12} \mathrm{GeV}\right)^{3 / 2}}{\left(g_{*} / 10^{2}\right)^{1 / 4}} .
$$

We have not yet computed the asymmetry $L$. As we will see the requirement of a large enough $L$ sets a lower bound on $\tilde{N}_{o}$, the initial amplitude of the $\tilde{N}_{1}$ oscillations. We will initially consider the case when $\rho_{\psi}>\rho_{\tilde{N}}$ at $t=\Gamma_{\tilde{N}}^{-1}$, which implies that (the decay product of) the inflaton dominates the energy density at all previous times (see Fig. 1). This sets an upper bound on $\tilde{N}_{o}$ and the viability of this variation of our model depends on the existence on an allowed interval for $\tilde{N}_{o}$.

Defining $\epsilon$ as the net lepton number per $\tilde{N}_{1}$ particle at decay, the total lepton number density at $t=\Gamma_{\tilde{N}_{1}}^{-1}$ is $n_{L} \simeq \epsilon n_{\tilde{N}_{1}}\left(t=\Gamma_{\tilde{N}_{1}}^{-1}\right) \simeq \epsilon \rho_{\tilde{N}}\left(t=\Gamma_{\tilde{N}}^{-1}\right) / M_{1}$, where $\rho_{\tilde{N}}$ redshifted as matter from the moment $\tilde{N}_{1}$ oscillations started, i.e. $t=M_{1}^{-1}$, when $\rho_{\tilde{N}}=M_{1}^{2} \tilde{N}_{o}^{2}$ (recall that we assume the $\tilde{N}_{1}$ potential is dominated by the quadratic term). Thus,

$$
n_{L} \simeq \epsilon M_{1} \tilde{N}_{o}^{2}\left[\frac{a\left(t=M_{1}^{-1}\right)}{a\left(t=\Gamma_{\tilde{N}}^{-1}\right)}\right]^{3}=\frac{\epsilon \tilde{N}_{o}^{2} \Gamma_{\psi}^{1 / 2} \Gamma_{\tilde{N}}^{3 / 2}}{M_{1}}
$$

In obtaining Eq. (7) it is necessary to remember that the Universe goes from MD to RD at $t=\Gamma_{\psi}^{-1}$, which occurs between $t=M_{1}^{-1}$ and $t=\Gamma_{\tilde{N}}^{-1}$. The entropy density at $t=\Gamma_{\tilde{N}}^{-1}$ 
is $s \simeq 4\left[\rho_{\psi}\left(\Gamma_{\tilde{N}}^{-1}\right)\right]^{3 / 4}$ and, since $\rho_{\psi}$ dominates the energy density of the Universe, we can equal $\rho_{\psi}$ to the critical density at the time, i.e. $\rho_{\psi}=(3 / 8 \pi) M_{P}^{2}\left(\Gamma_{\tilde{N}}^{2} / 4\right)$. We obtain

$$
L=\frac{n_{L}}{s} \simeq \frac{3.5 \epsilon \tilde{N}_{o}^{2} \Gamma_{\psi}^{1 / 2}}{M_{1} M_{P}^{3 / 2}} \simeq \frac{3.5 \epsilon \tilde{N}_{o}^{2} m_{\psi}^{3 / 2}}{M_{1} M_{P}^{5 / 2}} .
$$

The condition $L>L_{C}$ translates into

$$
\tilde{N}_{o}>0.5410^{17} \mathrm{GeV}\left(\frac{L_{C}}{0.1 \epsilon}\right)^{1 / 2} \frac{\left(M_{1} / 10^{4} \mathrm{GeV}\right)^{1 / 2}}{\left(m_{\psi} / 10^{11} \mathrm{GeV}\right)^{3 / 4}} .
$$

On the other hand $\rho_{\psi} / \rho_{\tilde{N}} \simeq 0.03\left(M_{P} / \tilde{N}_{o}\right)^{2}\left(\Gamma_{\tilde{N}} / \Gamma_{\psi}\right)^{1 / 2}$ at $t=\Gamma_{\tilde{N}}^{-1}$, and requesting $\rho_{\psi} / \rho_{\tilde{N}}>1$ yields

$$
\tilde{N}_{o}<0.75 \times 10^{17} \mathrm{GeV}\left(m_{\nu_{1}} / 10^{-4} \mathrm{eV}\right)^{1 / 4} \frac{\left(M_{1} / 10^{4} \mathrm{GeV}\right)^{1 / 2}}{\left(m_{\psi} / 10^{11} \mathrm{GeV}\right)^{3 / 4}} .
$$

This condition translates into an upper bound on $L$

$$
L<0.13 \epsilon\left(m_{\nu_{1}} / 10^{-4} \mathrm{eV}\right)^{1 / 2}
$$

thus $L>L_{C}$ only if $\epsilon>7 L_{C}\left(10^{-4} \mathrm{eV} / m_{\nu_{1}}\right)^{1 / 2}$. If $m_{\nu_{1}} \simeq 10^{-4} \mathrm{eV}$, then $\epsilon$ needs to be near maximum, $\epsilon \gtrsim 10^{-1}$. Let us see now that these large values of epsilon are easily obtainable in our scenario.

There are two generic mechanisms to obtain a non-zero $\epsilon$, i.e. a net lepton number per $\tilde{N}$ decay: one is to generate $\epsilon$ during the decay, due to $\mathrm{CP}$ and $L$ violation in $\tilde{N}$ decay modes, starting from an $L=0, \tilde{N}$ condensate, another is the generation of an $L$ asymmetric condensate, due to $\mathrm{CP}$ and $L$ violating effective operators. In the MSSM model supplemented with three right-handed neutrinos $\left(N_{i}, i=1,2,3\right)$ the generation of $\epsilon$ in the $\tilde{N}$ decay has been studied in the literature. If $M_{3}=M_{1}$, then $\epsilon \simeq \ln (2) \operatorname{Im}\left(h_{33}^{2}\right) / 8 \pi$ [18 could be as large as $10^{-2}$, otherwise there is a suppression factor $\simeq M_{1} / M_{3}$ [19. Here $h_{33}$ is assumed to be the largest $h_{i j}$ coupling in Eq. (3), and $\operatorname{Im}\left(h_{33}^{2}\right)$ could be as large as 1. However, we prefer not to impose any lower bound on the $\nu_{3}$ mass, thus leaving open the possibility of $M_{3}>>M_{1}$. 
This leaves us only the second method to account for the large $\epsilon$ needed. In this case, the decay of $\tilde{N}_{1}$, releases the lepton-number asymmetry accumulated in the condensate due to the appearance of effective lepton number violating soft supersymmetry breaking terms. These operators are due to the usual mechanism of supersymmetry breaking in the MSSM, i.e. supergravity breaking in a hidden sector. The main term of this type in our model is $O \simeq m_{3 / 2} M_{1} \tilde{N}_{1} \tilde{N}_{1}$, which yields a lepton number per $\tilde{N}_{1}$ particle

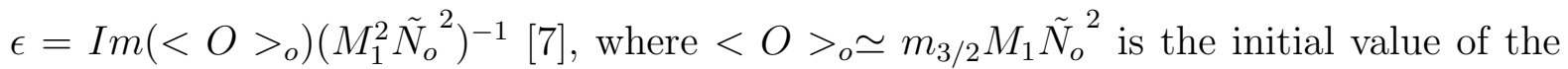
$L$-violating operator, thus, assuming $\operatorname{Im}\left(<O>_{o}\right) \simeq<O>_{o}$ we obtain $\epsilon \simeq m_{3 / 2} M_{1}^{-1}$. Replacing $m_{3 / 2} \simeq 1 \mathrm{TeV}$, we see that $\epsilon \gtrsim 10^{-1}$ is possible if $M_{1}<10 \mathrm{TeV}$, which is allowed by Eq (5), even if $m_{\nu_{1}} \simeq 10^{-4} \mathrm{eV}$ (recall that $m_{\psi}$ can be as low as $10^{11} \mathrm{GeV}$, a possibility in favor of which we argued above). Actually, the value of $\epsilon$ obtained is in general higher than this estimate because at the beginning of the oscillations the effective value of $m_{3 / 2}$ is $O(H)$, rather than $O(1 \mathrm{TeV})$, due to the supersymmetry breaking triggered by the non-zero vacuum energy (see below). Consequently, $\langle O\rangle_{o}$ is much larger, an thus the $L$ generated. Actually, a value $\epsilon \simeq O(1)$ may be available, even for $M_{1}<10 \mathrm{TeV}$. So, the previous estimate $\epsilon \simeq O(1 \mathrm{TeV}) M_{1}^{-1}$, represents a lower bound on $\epsilon$ rather than a precise value.

We see in Eq. (11) that even with $\epsilon \simeq 1, L$ can be up to $O(10) L_{C}$ but not much larger, unless $m_{\nu_{1}}>10^{-4} \mathrm{eV}$. We have so far kept $m_{\nu_{1}} \simeq 10^{-4} \mathrm{eV}$ because this value can easily accommodate the MSW solution to the solar neutrino problem. Since the mass square difference between $\nu_{1}$ and $\nu_{2}$ needs to be $\Delta m^{2} \simeq 10^{-6} \mathrm{eV}^{2}$ it is enough to choose $m_{\nu_{1}}<m_{\nu_{2}} \simeq 10^{-3} \mathrm{eV}$. However many models including this solution, mainly those trying to accommodate simultaneously various of the hints for non-zero neutrino masses [20], propose much larger $m_{\nu}$ values, such as $m_{\nu_{1}} \simeq O(\mathrm{eV})$. In this case the two oscillating neutrinos are almost degenerate, $m_{\nu_{1}} \simeq m_{\nu_{2}}$. This is the scenario we would need to accept in order to relax numerically the bound from Eq. (10) by one order of magnitude, 
i.e. for $m_{\nu_{1}}=1 \mathrm{eV}$, keeping $M_{1}$ and $m_{\psi}$ the same, we get from Eq.(10)

$$
\tilde{N}_{o}<0.75 \times 10^{18} \mathrm{GeV}\left(m_{\nu_{1}} / 1 \mathrm{eV}\right)^{1 / 4} \frac{\left(M_{1} / 10^{4} \mathrm{GeV}\right)^{1 / 2}}{\left(m_{\psi} / 10^{11} \mathrm{GeV}\right)^{3 / 4}} .
$$

In this case, from Eq (11) we see that $L<13 \epsilon$ with $m_{\nu_{1}} \simeq 1 \mathrm{eV}$, so $L$ could easily be up to $10^{2} L_{C}$.

We see that the best solutions, those with larger $L$, tend to saturate the upper bounds in Eqs.(10) and (11) so that at $t=\Gamma_{\tilde{N}}^{-1}$ we have $\rho_{\tilde{N}} \simeq \rho_{\psi}$. This leads us to the second variation of our model, namely to relax the condition of $\psi$-dominance, that yield Eqs. (10) or $(12)$, and allow $\rho_{\tilde{N}}$ to become larger than $\rho_{\psi}$ in the period, after $t=M_{1}^{-1}$, in which $\rho_{\tilde{N}}$ behaves like matter $\left(\tilde{N}_{1}\right.$ is oscillating) and $\rho_{\psi}$ (actually the density of their decay products) behaves like radiation.

Requesting the moment of equality, $t_{e q}$, namely the moment at which $\rho_{\tilde{N}}\left(t_{e q}\right)=$ $\rho_{\text {critical }}$, to happen before the $\tilde{N}$ decay, that is $t_{e q} \simeq 0.5\left(M_{P} / \tilde{N}_{o}\right)^{4} \Gamma_{\psi}^{-1}<\Gamma_{\tilde{N}}^{-1}$, reverses the inequality in Eq. (10) (and Eq. (12)), into an upper bound on $\tilde{N}_{o}$

$$
\tilde{N}_{o}>0.75 \times 10^{17} \mathrm{GeV}\left(\frac{m_{\nu_{1}}}{10^{-4} \mathrm{eV}}\right)^{1 / 4}{\frac{\left(M / 10^{4} \mathrm{GeV}\right)}{\left(m_{\psi} / 10^{11} \mathrm{GeV}\right)}}^{3 / 4} .
$$

In this case the s-neutrino decay is responsible for both, the production of the lepton asymmetry in fermions and the reheating of the Universe. Since $n_{L}=\epsilon \rho_{\tilde{N}}\left(t=\Gamma_{\tilde{N}}^{-1}\right) / M_{1}$ and $s=4\left[\rho_{\tilde{N}}\left(t=\Gamma_{\tilde{N}}^{-1}\right)\right]^{3 / 4}$, we obtain

$$
L=\frac{n_{L}}{s} \simeq \frac{\epsilon}{4 M_{1}}\left(\rho_{\tilde{N}}\left(\Gamma_{N}^{-1}\right)\right)^{1 / 4} \simeq 0.1 \epsilon \frac{\sqrt{M_{P} \Gamma_{\tilde{N}}}}{M_{1}} .
$$

In obtaining this equation we need to notice that, while at the beginning of the $\tilde{N}_{1}$ oscillations we still have $\rho_{\tilde{N}}=M^{2} \tilde{N}_{o}^{2}$, as before (see Eq. $(7)$ ), now in the redshift factor $\left[a\left(t=M^{-1}\right) / a\left(t=\Gamma_{\tilde{N}}^{-1}\right)\right]^{3}$ we have to take into account that the Universe is radiation dominated between $t \simeq \Gamma_{\psi}^{-1}$ and $t_{e q}$, and matter dominated before and after (until $\tilde{N}_{1}$ decay), see Fig. 1. Substituting, $\Gamma_{\tilde{N}} \simeq m_{\nu_{1}} M_{1}^{2} / 8 \pi v_{u}^{2}$ we get

$$
L=0.2 \epsilon\left(\frac{m_{\nu_{1}}}{10^{-4} \mathrm{eV}}\right)^{1 / 2} .
$$


This $L$ is about the maximum allowed in the previous scenario, Eq (11). Eq. (15) shows that $L$ can be larger for larger values of $m_{\nu_{1}}$. On the other hand, too large values of $m_{\nu_{1}}$ may lead to erasure of $L$. In order to see this point, let us consider the thermalization in this scenario. Here, the thermalization occurs instantaneously after the $\tilde{N}$ decay, because the rate of interaction of the decay products $\Gamma_{i n t}=P n_{\tilde{N}} \sigma=P^{3} n_{\tilde{N}} \alpha^{2} M_{1}^{-2}$ at the moment of decay, is larger than the expansion rate of the Universe at that time, $H \simeq \Gamma_{\tilde{N}}$,

$$
\frac{\Gamma_{i n t}}{\Gamma_{\tilde{N}}} \simeq \frac{\alpha^{2}}{48 \pi^{2}} P^{3} \frac{M_{P}^{2}}{v_{u}^{2}} \frac{m_{\nu_{1}}}{M_{1}} \simeq 2 \times 10^{11}\left(\frac{\alpha}{100}\right)^{2}\left(\frac{P}{2}\right)^{3}\left(\frac{m_{\nu_{1}}}{10^{-4} \mathrm{eV}}\right)\left(\frac{10^{4} \mathrm{GeV}}{M_{1}}\right) .
$$

Thus, the $\tilde{N}$ energy density is immediately thermalized after the decay, $\rho_{\tilde{N}}\left(\Gamma_{\tilde{N}}^{-1}\right)=$ $(3 / 8 \pi) M_{P}^{2} \Gamma_{\tilde{N}}^{2}=\left(g_{*} / 3\right) T_{t h}^{4}$, and

$$
T_{t h} \simeq \frac{M_{1} \sqrt{M_{P} m_{\nu_{1}}}}{\left(30 \pi^{3} g_{*}\right)^{1 / 4} v_{u}} \simeq \frac{5 \times 10^{3} \mathrm{GeV}}{\left(g_{*} / 100\right)^{1 / 4}}\left(\frac{M_{1}}{10^{4} \mathrm{GeV}}\right)\left(\frac{m_{\nu_{1}}}{10^{-4} \mathrm{eV}}\right)^{1 / 2} .
$$

Notice that $T_{t h}<M_{1}$ if $m_{\nu_{1}}<4 \times 10^{-4} \mathrm{eV}$, thus the sneutrinos $\tilde{N}_{1}$ are not present in the thermal bath. For larger values of $m_{\nu_{1}}, T_{t h}>M_{1}$ and one should worry about the erasure of $L$ caused by the decay of the $\tilde{N}_{1}$ present in the thermal bath. Even if the $L$ initially generated could be as large as $10^{2} L_{C}$, with $m_{\nu_{1}} \simeq 1 \mathrm{eV}$ this erasure could lower $L$ considerably. If we do not want to worry about this source of $L$-erasure, the value of $m_{\nu_{1}}$ is fixed at $m_{\nu_{1}} \simeq 10^{-4} \mathrm{eV}$, since it cannot be smaller for $L \gtrsim L_{C}$, Eq. (15).

Notice that in the first variation of our scenario the preferred neutrino masses are of order $\mathrm{eV}$ or larger. Without any major modification of our model we could choose $\nu_{1}$ to be heaviest of the three light neutrinos. In this case the lightest right handed neutrino would mix in the mass matrix preferably with the heaviest light neutrino and not the lightest, in a see-saw scenario with $m_{\nu_{1}}^{D} \simeq m_{\nu_{2}}^{D} \simeq m_{\nu_{3}}^{D}$. Doing so the two lightest neutrino masses are unconstrained in our model. We could do the same thing in the second variation of our scenario, but in this case the preferred value of the heaviest neutrino would be $10^{-4}$ $\mathrm{eV}$. This would not be compatible with the heaviest neutrino accounting for part of the dark matter, for example something totally possible in the first variation. 
Let us address the issue of how the necessary $\tilde{N}_{0}$ could be generated. We have seen that in the first scenario we considered that $\tilde{N}_{0}$ has to be between $10^{17}$ and $10^{18} \mathrm{GeV}$, while in the second scenario we need $\tilde{N}_{0}>10^{17} \mathrm{GeV}$. There are several mechanisms that can produce a value of $\tilde{N}_{o} \simeq 10^{-2} M_{P}$ or larger. Let us recall that during inflation supersymmetry is necessarily broken since the energy density $V_{o}$ is non zero. This induces a mass proportional to the Hubble parameter $H$ for all flat directions, thus $m_{\tilde{N}_{1}}^{2}=$ $c H^{2}+M^{2}$. Dine et al. 221 assumed that $c$ is negative and of order one, and that non-renormalizable terms $\delta W \simeq \lambda \tilde{N}_{1}^{n} /\left(n\left(\beta M_{P}\right)^{n-3}\right)$ in the superpotential stabilize the minimum to the value

$$
\tilde{N}_{o}=\left(\frac{\sqrt{-c} H\left(\beta M_{P}\right)^{n-3}}{(n-1) \lambda}\right)^{1 /(n-2)} .
$$

It is obvious that $H<\tilde{N}_{o}<M_{P}$ (with the constants $\beta$ and $c$ of order one) and $\tilde{N}_{o}$ becomes arbitrarily closer to $\beta M_{P}$ for large $n$. The conditions to obtain the necessary negative effective masses and non-renormalizable terms to lift the "flat" direction of the potential in supergravity scenarios have in general been examined in Ref. [22], both in D- and F- inflationary models. Large negative masses square favour F-inflationary scenarios in which the Kähler potential contains a large mixing of the sneutrino and the inflaton in the term quadratic in $N_{1}$. This is a strong constraint on supergravity scenarios, which, for example, excludes minimal supergravity and in string-based models requires that the inflaton be a $T$ modulus field (and not the dilaton $S$ ). In particular, in orbifold constructions, $m_{\tilde{N}_{1}}^{2} \leq 0$ at the origin, is obtained if the modular weight of the $N_{1}$ field is $n_{\tilde{N}_{1}} \leq-3$.

There is another possibility we think cannot be neglected, namely that $c$ is very small. The value $m_{\tilde{N}_{1}}^{2} \simeq 0$ at the origin can also appear in a natural way, with no fine-tuning at all, both in $F$-inflation and in $D$-inflationary scenarios [22]. In this case, $\tilde{N}_{o}$ departs from zero due to quantum fluctuations during the Sitter phase. The coherence length of 
these fluctuations $\ell_{c o h} \simeq H_{i n f}^{-1} \exp \left(3 H_{\text {inf }}^{2} / 2 m^{2}\right) \simeq H_{i n f}^{-1} \exp (3 / 2 c)$ would be large enough to encompass our present visible Universe if $c<2 \times 10^{-2}$. When the coherence length is much larger than the horizon, $\ell_{c o h} \gg H_{\text {inf }}^{-1}$, as is the case here, the field $\tilde{N}_{1}$ can be treated as classical and

$$
\tilde{N}_{o}=\sqrt{\left\langle\tilde{N}_{1}^{2}\right\rangle} \simeq \sqrt{\frac{3}{8 \pi^{2}}} \quad \frac{H_{i n f}^{2}}{M}=\sqrt{\frac{3}{8 \pi^{2}}} \quad \frac{H_{i n f}}{\sqrt{c}} .
$$

For $\tilde{N}_{o} \simeq 10^{17} \mathrm{GeV}$ and $H_{\text {inf }} \simeq 10^{13} \mathrm{GeV}$ we need $c \simeq 4 \times 10^{-10}$. This small value of $c$ can be naturally achieved in string-based $F$-inflationary models. In $T$-driven models with orbifold compactifications this possibility is insured by the discrete character of the modular weights, since it is enough that $n_{\tilde{N}_{1}}=-3$. In $D$-inflationary models, usually the $D$-term is associated to an anomalous $\mathrm{U}(1)$, and $m_{\tilde{N}_{1}}^{2} \simeq 0$ results if the $N_{1}$ field is a singlet under that group [22].

In conclusion, we presented here a model to produce a very large lepton asymmetry $L \simeq 10^{-2}-1$ without producing a large baryon asymmetry. The model is based on the Affleck and Dyne scenario, in which the field acquiring a large vacuum expectation value during inflation is an sneutrino right. We take as a specific model the MSSM supplemented with three right handed neutrino singlet superfields, besides an inflationary sector. We considered two variations of one scenario, one in which the inflaton energy dominates at reheating and another in which the sneutrino energy dominates then. In both cases we required $L$ to be large enough for the electroweak symmetry to be spontaneously broken at all temperatures after inflation, with the consequent suppression of sphaleron transitions. In order to obtain this, in our scenario the large $L$ is generated before thermalization. The models considered are realistic. The first variation works better if the lightest neutrino mass is of $O(1 \mathrm{eV})$. In this case the MSW solution to the solar neutrino problem would require almost degenerate neutrinos, as proposed in models trying to account simultaneously for several of the present hints for non-zero neutrinos 
masses. Alternatively, if the bounds apply to the heaviest neutrino instead, the lighter neutrino masses are unconstrained. The second variation works better when the lightest

neutrino mass is of $O\left(10^{-4}\right) \mathrm{eV}$, what can easily accommodate the masses needed for the MSW mechanism. In both cases the preferred value of the lightest right handed neutrino is of $O(\mathrm{TeV})$ and the vacuum expectation value of the s-neutrino field during inflation must be larger than $10^{17} \mathrm{GeV}$. We also commented on ways to obtain naturally these large values for the sneutrino condensate. We have not addressed here how the baryon asymmetry could be generated. A very interesting possibility that deserved further study is that the out of equilibrium sphaleron transitions may translate a minor part of the $L$ asymmetry into $B$ [10.

\section{Acknowledgements}

We thank the Aspen Center for Physics, where this paper was initiated, for its hospitality.

\section{References}

[1] R. V. Wagoner, W. A. Fowler and F. Hoyle, Ap. J. 148 (1967) 3; A. Yahil and G. Beaudet, Ap. J. 206 (1976) 26; Y. David and H. Reeves, Physical Cosmology (ed. R. Balian, J. Audouze and D. N. Schramm, North-Holland, Amsterdam, 1980); J. N. Fry and C. J. Hogan, Phys. Rev. Lett. 49 (1982) 1783; R. J. Scherrer, Mon. Not. Roy. Astron. Soc. 205 683; N. Terazawa and K. Sato, Ap. J. 294 (1985) 9; K. Olive, D. N. Schramm, D. Thomas and T. Walker, Phys. Lett. B265 (1991) 239; G. Starkman Phys. Rev. D45 (1992) 476.

[2] H. Kang and G. Steigman, Nucl. Phys. B372 (1992) 494.

[3] K. Freese, E. W. Kolb and M. S. Turner, Phys. Rev. D27 (1983) 1689. 
[4] M. White, J. Navarro, A. Evrard and C. Frenk, Nature 366 (1993) 429.

[5] G Steigman and J. Felten, OSU-TA-24-94, astro-ph/9502029.

[6] J. A. Harvey and E. W. Kolb, Phys. Rev. D24 (1981) 2090.

[7] I. Affleck and M. Dine, Nucl. Phys. B249 (1985) 361.

[8] P. Langacker, G. Segre and S. Soni, Phys. Rev. D26 (1982) 3425.

[9] V. A. Kuzmin, V. A. Rubakov and M. E. Shaposnikov, Phys. Lett. B155 (1985) 36.

[10] J. Liu and G. Segrè, Phys. Lett. B338 (1994) 259.

[11] A. D. Dolgov and D. P. Kirilova, J. Moscow Phys. Soc. 1 (1991) 217 (see also A. D. Dolgov, IUPAP Conference on Primordial Nucleosynthesis and Evolution in the Early Universe, Tokyo, 1990); A. D. Dolgov, Phys. Rep. 222 (1992) 309.

[12] R. Foot, M. J. Thompson and R. R. Volkas, Phys. Rev D53 (1996) 5349; X. Shi, Phys. Rev. D54 (1996) 2753.

[13] A. D. Linde, Phys. Rev. D14 (1976) 3345.

[14] S. Davidson, H. Murayama and K. Olive, Phys. Lett. B328 (1994) 345.

[15] L. Kofman, A. D. Linde and A. A. Starobinsky, Phys. Rev. Lett. 73 (1994) 3195; Phys. Rev. Lett. 76 (1996) 1011.

[16] D. S. Salopek, Phys. Rev. Lett. 69 (1992) 3602; L. Krauss and M. White, Phys. Rev. Lett. 69 (1992) 869; A. Liddle and D. Lyth, Phys. Rep. 231 (1993) 1.

[17] G. G. Ross and S. Sarkar, Nucl. Phys. B461 (1996) 597. 
[18] H. Murayama, H. Suzuki, T. Yanagida and J. Yokoyama, Phys. Rev. Lett. 70 (1993) 1012.

[19] B. Campbell, S. Davidson and K. Olive, Nucl. Phys. B399 (1993) 111.

[20] D. Caldwell and R. Mohapatra, Phys. Rev. D48 (1993) 3259; J. T. Peltoniemi and J. W. F. Valle, Nucl. Phys. B406 (1993) 409.

[21] M. Dine, L. Randall and S. Thomas, Nucl. Phys. B458 (1996) 291.

[22] J. A. Casas and G. B. Gelmini, Phys. Lett. B410 (1997) 36. 


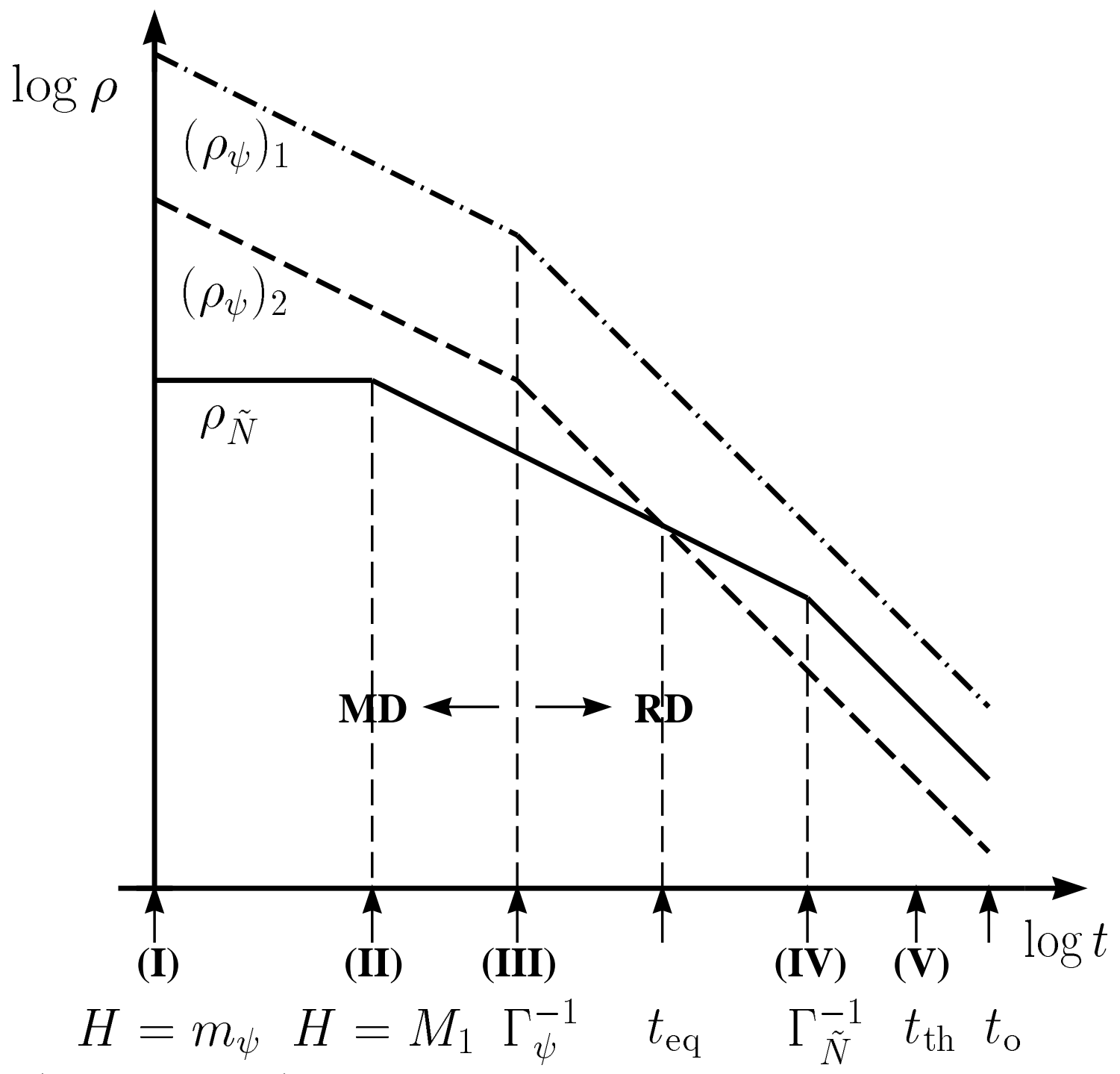

(end inflation)

Figure 1: Schematic view of the sequence of events we envision after the end of inflation. See the text for details. The dashed-dotted and dashed lines show the energy density of the inflaton (before III) and its decay products (after III) in the first and second variations of our scenario, respectively. The solid line shows the energy density of the relevant sneutrino and its decay product. The Universe is matter dominated (MD) before the inflaton decays (III) and in the first variation it is radiation dominated (RD) afterwards. In the second variation de Universe becomes again MD for a period before the sneutrino decays. 